\title{
Fast Handover Algorithm Based on DAD-Less Mechanism for Hierarchical Mobile IPv6
}

\author{
Jia-Bao Fang \\ China National Digital Switching System Engineering and \\ Technological Research Center \\ Zhengzhou 450002, China \\ 2014xdfjb@sina.com \\ Zhen-Xing Wang \\ China National Digital Switching System Engineering and \\ Technological Research Center \\ Zhengzhou 450002, China
}

\author{
Lian-Cheng Zhang \\ China National Digital Switching System Engineering and \\ Technological Research Center \\ Zhengzhou 450002, China
}

\begin{abstract}
A fast handover algorithm using DAD-Less mechanism for hierarchical mobile IPv6 was proposed for the problem of mobile node handover latency by duplicate address detection in HMIPv6 in order to improve the performance of handover for HMIPv6. The IP address allocation mechanism and new interface identifier were used to distribute unique interface identifier within a domain for the node in the mobile anchor point, ensuring the uniqueness of the link to address without the need for the duplicating address detection within the domain through the stateless auto-configuration mechanism configuration address. Performance analysis and the experimental results show that, compared with standard HMIPv6, algorithm of switching delay reduced by about $90 \%$, which is more suitable for frequent switching nodes.
\end{abstract}

Keywords-Hierarchical mobile IPv6; Handover latency; Duplicate address detection; Interface identifier

\section{INTRODUCTION}

In order to support mobility management in next-generation networks, Internet Engineering Task Force (IETF) began to develop Mobile IPv6 Protocol (MIPv6) in the end of last century [1]. At present, mobile IPv6 protocol promotes maneuverability of Mobile Node (MN) in internet and improves the performance of $\mathrm{MN}$ handover. However, the longer latency in Duplicate Address Detection (DAD) and banding update makes it unacceptable for some communication services which require higher Quality of Service (QoS). Therefore, scholars have put forward many improved methods, including Hierarchical Mobile IPv6 (HMIPv6)[2], which solved the problem that MIPv6 mobile node needs frequent registration at Home Agent (HA) and Correspondent Node $(\mathrm{CN})$ through the Internet hierarchy classification. But it still adopts the DAD working pattern of MIPv6, resulting in less obvious optimization of switching delay and even worse performance than MIPv6 in the process of MN handover in different Mobile Anchor Point(MAP)[3].
At present, HMIPv6 research mainly focuses on the choice of MAP and the analysis of layer division, etc. To be specific, the literature [4] combined Mobile IPv6 with Fast Handover (FMIPv6)[5] and HMIPv6 to propose Fast Handover for Hierarchical Mobile IPv6 (FHMIPv6); Literature [6] and literature [7] analyzed the influence of multi-layer HMIPv6 on handover latency by mathematics method and testbed respectively and pointed out that the three-layer-HMIPv6 is beneficial to mobile management.

Literature [8] analyzed performance advantages and disadvantages of FHMIPv6, HMIPv6, Proxy Mobile IPv6 (PMIPv6) and Fast Handover for Proxy Mobile IPv6 (FPMIPv6) through mathematical model; Literature [9] proposed a cascade tunnel mode in order to reduce handover latency and signaling load of HMIPv6; and literature [10] put forward metrics based on QoS preferences of MNs and QoS abilities of MAPs, in order to perform a simulative evaluation of the HMIPv6 network implementation with QoS-aware dynamic MAP selection.

However, the researches of DAD on HMIPv6 are very few. DAD mode of standard HMIPv6 requires too much time, which severely impacts $\mathrm{MN}$ handover performance. Literature [11] proposed an Optimized HMIPv6 (O-HMIPv6) algorithm, which is based on homogeneous prefix concept. The 64 bit IPv6 prefix is divided into two parts: 48 bit Network Identifier (NID) and 16 bit Subnet Identifier (SID). The HMIPv6 network is designed in the way that each AR of a domain adopts the same NID to ensure the uniqueness of LCoA within the domain so that $\mathrm{DAD}$ is unnecessary in the procedure of intra-domain handover and then the handover latency decrease is expected. However, the algorithm restricts the range of MAP domain, which is unbeneficial to the deployment. Literature [12] proposed a DAD-less MIPv6 mechanism which could completely remove the DAD process from the handover, but it need every MN with a unique IPv6 address on internet which isn't possible in present environment. 
A fast handover algorithm based on DAD-Less mechanism for HMIPv6 is proposed for the problem of mobile node handover latency by DAD in HMIPv6 in order to improve the performance of handover for HMIPv6. This algorithm redesigns the procedure of the address configuration in the MAP domain to ensure the uniqueness of Interface Identifier (IID) of the MN within the MAP domain so as to avoid the operation of DAD within the MAP intra-domain movement and improve HMIPv6 handover performance.

\section{FAST HANDOVER ALGORITHM}

This section describes the fast handover algorithm of A-DAD-HMIPv6, which sets up a new process of address configuration. This algorithm modifies the 64 bit IID structure to distinguish local address and the MN address; then, build the Hash table of MNs' IID in MAP to ensure the uniqueness of MNs' IID within MAP domain. Finally, achieve DAD-Less when MN moves within the same MAP.

To achieve the arithmetic of A-DAD-HMIPv6, some changes are required in the address structure of MIPv6, the configuration of address, and the MAP.

\section{A. Address Structure}

The DAD operation of IPv6 aims at ensuring the uniqueness of address in the net. By redrawing the 64-bit IPv6 address interface identifier to guarantee the uniqueness in intra-domain on MAP and avoid DAD after applying the address.

a) Address structure of standard HMIPv6

HMIPv6 uses the global unicast address. The structure of the address is shown in Fig. 1.

b) Address structure of optimization algorithm
Optimization algorithm is designed to ensure the uniqueness of the interface identifier, so it is supposed to modify IID, and the specific structure is shown in Fig. 2.

The algorithm is divided 64-bit interface identifier into two parts: A mobile flag and 63 interface ID. Mobile flag is used to distinguish local address and mobile address where 0 represents the local address, 1 represents mobile address. It will set mobile flag 0 in default in local address configuration procedure. When $\mathrm{MN}$ requests $\mathrm{LCoA}$ from the home agent or MAP, MAP or home agent will set mobile flag to the location 1 when it generates the mobile address. This can ensure that the local address does not conflict with the mobile address. The last 63 bits of the interface ID is used to distinguish the mobile address which is applied at the same MAP domain. This two-layer structure of the address can guarantee the uniqueness of the MNs' IID within the MAP domain.

\section{B. Address Configuration}

IPv6 address configuration process is divided into stateful auto-configuration and stateless auto-configuration. The A-DAD-HMIPv6 algorithm has already got a unique unicast address within the domain in the initial stage through stateless auto-configuration, so it doesn't require stateful auto-configuration.

a) Address configuration of standard HMIPv6

In standard HMIPv6 protocol, when the MN moves to a new network, MN is assigned a new LCoA. It generally uses the stateless auto-configuration to generate addresses, LCoA is combined with current network prefix and interface identifier of MN. As shown in Fig. 3, MN's IPv6 from 2001: 0db: 33: 1: A: B: C: D is changed to 2001: 0db: 32: 2: A: B: C: D. MN's movement changes the network prefix of IPv6 address and interface identifier has not changed.

\begin{tabular}{|c|c|c|}
\hline Global routing prefix & SubnetID & Interface ID \\
\hline 48 bit & $16 \mathrm{bit}$ & $64 \mathrm{bit}$ \\
\hline Common topology & Site topology & Interface identifier \\
\hline
\end{tabular}

Figure 1. IPv6 global unicast address structure.

\begin{tabular}{|c|c|c|c|}
\hline Global routing prefix & SubnetID & $\mathrm{M}$ & Interface ID \\
\hline 48bit & $16 \mathrm{bit}$ & $63 \mathrm{bit}$ & \\
\hline Common topology & Site topology & $\mathrm{M}$ : Mobile flag $\quad$ Interface identifier
\end{tabular}

Figure 2. Address structure of optimization algorithm. 


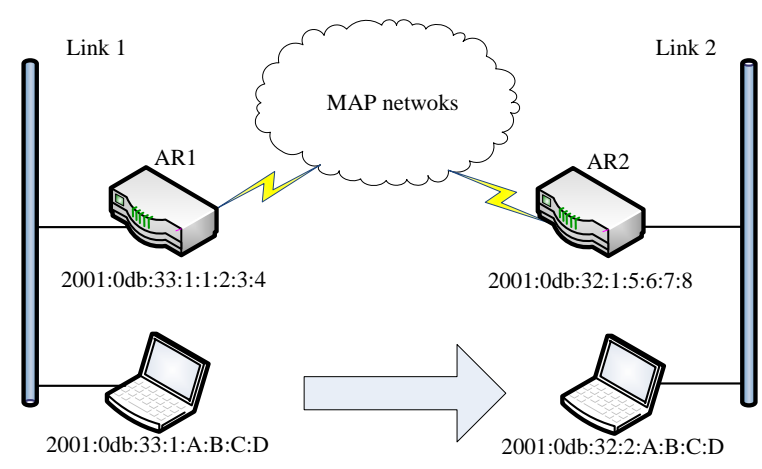

Figure 3. IP address handover of MN.

Then MN will use DAD to test whether LCoA is unique in the HMIPv6 protocol. If the current network has already had this address, the router will use stateful auto-configuration (DHCPv6) to generate a new address, then it will use DAD to test the address until generate a locally unique LCoA.

b) Address configuration of optimization algorithm

The address configuration of this algorithm only requires stateless auto-configuration. $\mathrm{MN}$ configures a MAP region unique interface identifier in the address configuration phase. Further, the intra-domain handover can guarantee the uniqueness of $\mathrm{MN}$ addresses and avoid DAD operation. Its address configuration steps are as follows:

1. Generate a random 64-bit value.

2. Its bit 1 and bit 7 is set to 1 . The first one corresponds to the mobile flag, set 1 as MN address. Bit 7 corresponds to $\mathrm{U} / \mathrm{L}$ bit, set 1 indicates a locally administered interface identifier. The result is the new IID. (If it is a local host, bit 1 is set to 0 , bit 7 is set to 1 to generate a new IID, then through standard IPv6 address configuration to generate a new IP address.)

3. The newly generated IID is sent to the MAP; MAP test its uniqueness by look-up hash tables; if the IID is unique, send routes announce information to $\mathrm{MN}$, and go to Step 4; If the IID is not unique, return to step 1 , regenerate the IID.

4. When MN gets the current subnet prefix, it uses stateless auto-configuration to generate LCoA and RCoA.

Via the new address configuration, $\mathrm{MN}$ obtains the LCoA and RCoA, thus its IID does not conflict with other nodes in the MAP domain. Therefore, when the MN moves in the MAP region, stateless auto-configuration always been able to guarantee its address does not conflict with other nodes, which means no further DAD operation.

\section{MAP Work}

MAP's function may act as "local" home agent to MN; its role is similar to the $\mathrm{MN}$ home agent.

The role of MAP in optimization algorithm requires further expansion. The IID of MN needs to be tested whether it is repeated to ensure its uniqueness in the MAP region. In the end, MAP needs to maintain a hash table of MN address to rapidly test the MN's new address IID. Specific methods are as follows:

1. MAP receives message from MN, gets MN's IID.

MAP tests IID of MN by look-up table. If repeated, send address validation failure message to the $\mathrm{MN}$; If not, through the hash algorithm add MN address to the hash table, and then send the address test success message to the MN. The hash algorithm uses division method, takes the last 16 bits of IID as the hash value, and the algorithm is as follows:

$$
H(I I D)=I I D \bmod 2^{16}
$$

2. Using linear probing hash as a collision avoidance algorithm:

3. MAP establishes MN's address table shown as follows:

\section{New Messages and Options}

Optimization algorithm changes DAD mode of HMIPv6. Communications need to add some new options and message between MN and MAP.

a) New ICMPv6 packets

It includes detection solicitation (DS) and detection acknowledgement (DA) to test the IID of MN address whether repeated. Wherein, DS sent by MN to MAP is used to initiate a test request and DA replies to the $\mathrm{MN}$ after MAP completion of the inspection operation. Optimization algorithm designs the new message as shown in Fig. 4.

Type field value 150 , indicates that the packet belongs to IPv6 type. Checksum field is the message header checksum. Subtype field is used to distinguish between DA and DS, DS corresponding to a value of 10, DA corresponding to a value of 11 . Code field is used to indicate status. In DS, the default of the field is 0; For DA, this field reflects the final result of the procedure to test IID:

0 indicates success;

128 indicates that the operation fails and IID repeats;

129 indicates the appearance of an error during the operation;

$$
H_{i}=\left(H(I I D) \pm d_{i}\right) \bmod 2^{16}, i=1,2, \ldots, k\left(k \leq 2^{16}\right), d_{i}=1,2,3, \ldots, 2^{16}-1
$$

TABLE 1. MN ADDRESS HASH TABLE.

\begin{tabular}{llllll}
\hline Sequence number & IID & RCoA & HoA & Lifetime & Flag \\
\hline 1 & $4: 3: 2: 1$ & $2001: 0 \mathrm{db}: 32: 2: 4: 3: 2: 1$ & $2001: 0654: 34: 5: 12 \mathrm{ac}: 3: 215: 5$ & 250 & $\mathrm{~A} / \mathrm{H} / \mathrm{K} / \mathrm{L}$ \\
2 & $5: 4: 3: 2$ & $2001: 035 \mathrm{a}: 15: 3: 5: 4: 3: 2$ & $2001: 021 \mathrm{~b}: \mathrm{a} 453: 5: 123: 356: 2: 1$ & 250 & $\mathrm{~A} / \mathrm{H} / \mathrm{L}$ \\
\hline
\end{tabular}


130 represents that MAP does not support this operation.

Identifier field can promote the MN's judgment whether DA can match the DS which is sent in prior. IID field is used to store the IID.

$0 \quad$ 1
01234567890123456789012345678901
\begin{tabular}{|c|c|c|}
\hline Type & Code & Checksum \\
\hline Subtype & Reserved & Identifier \\
\hline \multicolumn{3}{c}{ IID } \\
\hline
\end{tabular}

Figure 4. New message format.

b) New RCoA options

During the operation of DAD in RCoA, the LBU and LBA of HMIPv6 packets are still adopted, as shown in Fig. 5. The LBA adds a new option - RCoA Option, as shown in Fig. 6. If MAP updated RCoA, the MAP must contain the RCoA option in LBA to reply MN, the LBA's State field need to define a new status value (range 2 to 127) to inform $\mathrm{MN}$ the new RCoA.

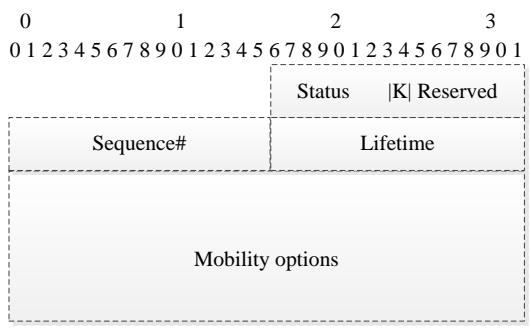

Figure 5. LBA packet format.

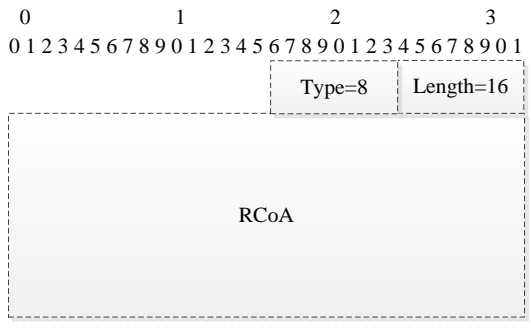

Figure 6. New RCoA options.

\section{E. The Overall Process of the Algorithm Description}

When the MN moves to a new MAP domain, it will use stateless auto-configuration which combines IID with network prefix from router advertisement packets, and then it will generate $\mathrm{LCoA}$ and $\mathrm{RCoA}$, finally $\mathrm{MN}$ will apply DAD to detect them.

a) LCoA DAD

If the MN moves from the home link to a new MAP domain, firstly it will generate a new format of IID, then it will send DS message to MAP. If it moves from a MAP domain to a new one, then it will send DS packets directly to the MAP domain. Wherein, IID in DS packets is being used in MN. After MAP receiving the DS packet sent by $\mathrm{MN}$, it will detect the MN's IID in the hash table of MN address by hashing algorithm, If there is the same IID, then it will send DA packet of operation failed to $\mathrm{MN}$, requesting $\mathrm{MN}$ to reconfigure IID; If there isn't the same IID, it will send the DA packets of successful operation to the MN, MN will directly use LCoA and RCoA which are generated by stateless auto-configuration. RCoA and LCoA are same if MN moves to a new MAP domain, so it needn't DAD operation again.

b) MAP registration

When the MN moves in a new MAP domain, it will use stateless auto-configuration. According to this provision, it should ensure the uniqueness of MN's IID in MAP domain, and the MAP domain operation can guarantee the uniqueness of LCoA, and thus MN does not need DAD. After the MN generates LCoA, it also needs to register on MAP, the steps should be consistent with HMIPv6.

From the above part, A-DAD-HMIPv6 algorithm uses previously lookup mechanism and modifications IID structure to ensure the uniqueness of the MN within the MAP domain. It realizes LCoA and RCoA's address configuration process to avoid DAD, effectively reducing the delay of HMIPv6 domain handover.

\section{PERFORMANCE ANALYSIS OF AlgORITHMS}

In order to analyze handover procedure effectively, mathematical analysis will be used to analyze the main parameters of MN handover procedure. Table 2 describes the main parameters of the handover process and the meaning it represents.

TABLE 2. DESCRIPTION OF PARAMETERS.

\begin{tabular}{ll}
\hline Parameters & Description \\
\hline$E[B i]$ & The average signal interval \\
$E[R A]$ & average interval of route advertisement \\
$t_{\alpha}$ & Transmission time between MN\&AP \\
$t_{\beta}$ & Transmission time between AR\&AP \\
$t_{h}$ & Once transmission time of wired jump \\
$t_{D A D}$ & DAD time \\
$t_{\text {look }} H T$ & Time of lookup Hash table \\
$x$ & Hops between AR and MAP \\
$y$ & Hops between HA and MAP \\
$z$ & Hops between HA and CN \\
$w$ & Hops between CN and MAP \\
\hline
\end{tabular}

Handover latency formulas for HMIPv6 and A-DAD-HMIPv6 (using A-DAD-H represents in formula) domains macro mobility are as follows:

$$
\begin{gathered}
T_{\text {int } e r}^{H M I P \nu 6}=d_{L 2}+d_{M D+L_{-} C O N F}+d_{R_{-} C O N F}+d_{L B U}+d_{G B U} \\
T_{\text {inter }}^{A-D A D-H}=d_{L 2+R_{-} C O N F}+d_{L B U}+d_{G B U}
\end{gathered}
$$


Among them, $d_{L 2}$ represents a delay of the data link layer, it includes the signal response, certification process and associated node. $d_{M D+L_{-} C O N F}$ represents a delay of network layer movement detection and LCoA configuration process. $d_{R_{-} \text {CONF }}$ represents a delay of RCoA configuration process. $d_{L B U}$ is the handover delay of LBU and LBA. $d_{L B U}$ indicates handover delay of GBU and GBA between $\mathrm{MN}$ and $\mathrm{CN}$, which contains the return routing testing. GBU and GBA exchange between $\mathrm{MN}$ and $\mathrm{HA}$, which is hidden in the return routing tests, invisible in the macro move of domain. The calculation formula is as follows:

$$
\begin{gathered}
d_{L 2}=E[B i]+t_{\alpha} \\
d_{M D+L_{-} C O N F}=E[R A]+t_{\alpha}+t_{D A D}+t_{\beta} \\
d_{R_{-} C O N F}=2\left(t_{\alpha}+t_{\beta}+x \cdot t_{h}\right)+t_{D A D} \\
d_{L B U}=2\left(t_{\alpha}+t_{\beta}+x \cdot t_{h}\right) \\
d_{G B U}=2\left(t_{\alpha}+t_{\beta}+(x+y+z) \cdot t_{h}\right)+2\left(t_{\alpha}+t_{\beta}+(x+w) \cdot t_{h}\right) \\
d_{L 2+R_{-} C O N F}=E[B i]+t_{\alpha}+2 \cdot\left(t_{\beta}+x \cdot t_{h}\right)+t_{\text {look }-H T}
\end{gathered}
$$

The formula of handover delay required by HMIPv6 and A-DAD-PMIPv6 domain micro mobility is:

$$
\begin{gathered}
T_{\text {int } r a}^{H M M P v 6}=d_{L 2}+d_{M D+L_{-} C O N F}+d_{L B U} \\
T_{\text {int } r a}^{A-D A-H}=E[B i]+t_{\alpha}+2 \cdot\left(t_{\beta}+x \cdot t_{h}\right)+d_{L B U}
\end{gathered}
$$

Parameter values are calculated by the formula (5), (6) and (8). The A-DAD-HMIPv6 algorithm only includes signal response and LBU handover time.

MN maintains the overall delay depends on the frequency of Inter-domain macro mobility and micro mobility domain during the session. The overall handover delay can be calculated as follows, where $E\left[H^{M}\right]$ and $E\left[H^{C}\right]$ represent frequency of inter-domain handover and frequency of intra-domain handover.

$$
\begin{gathered}
T_{\text {total }}^{H M I P v 6}=E\left[H^{M}\right] \cdot T_{\mathrm{inter}}^{H M I P v 6}+\left(E\left[H^{C}\right]-E\left[H^{M}\right]\right) \cdot T_{\mathrm{int} r a}^{H M P_{\nu} 6} \\
T_{\text {total }}^{A-D A D-H}=E\left[H^{M}\right] \cdot T_{\mathrm{inter}}^{A-D A D-H}+\left(E\left[H^{C}\right]-E\left[H^{M}\right]\right) \cdot T_{\mathrm{int} r a}^{A-D A D-H}
\end{gathered}
$$

The experiments simulated HMIPv6 and A-DAD-HMIPv6 algorithm and obtained handover delay in these two cases of MN. According to literature [13], the main parameters of the experiment is set to: $E[B i]=50 \mathrm{~ms}$ $E[R A]=0.5 \mathrm{~s}, t_{\alpha}=10 \mathrm{~ms}, t_{\beta}=1 \mathrm{~ms}, t_{h}=1 \mathrm{~ms}, t_{D A D}=1 \mathrm{~s}$, $t_{\text {look } \_ \text {HT }}=20 \mathrm{~ms}, x=6, y=20, z=20, w=30$.

When the MN only moves in the MAP region, the relationship between total delay and handover frequency of $\mathrm{MN}$ is shown in Fig. 7. When the MN moves between MAP domains, experiment sets that each inter-domain movement will have twice movements in the domain. The relationship between the total delay and handover frequency inter-domain of MN is shown in Fig. 8.

By analyzing the results we found, for both Micro mobility domain and inter-domain macro mobility, A-DAD-HMIPv6 handover latency are significantly lower than HMIPv6; And with the increase of inter-domain and intra-domain handover frequency, HMIPv6 handover latency increases rapidly, but the handover latency of A-DAD-HMIPv6 is able to maintain a low level. Obtained by the mathematical way, A-DAD-HMIPv6 and HMIPv6's handover latency are both proportional with mobile frequencies. Fig. 7 and Fig. 8 show that the total delay contrasts with HMIPv6 and A-DAD-HMIPv6 in intra-domain and inter-domain handover, clearly demonstrating the effect of optimizing the A-DAD-HMIPv6. The handover latency caused by HMIPv6 is 10 times about A-DAD-HMIPv6. This means that A-DAD-HMIPv6 works more efficiently in frequent handover of mobile environment, so it can better meet the demand for high QoS services.

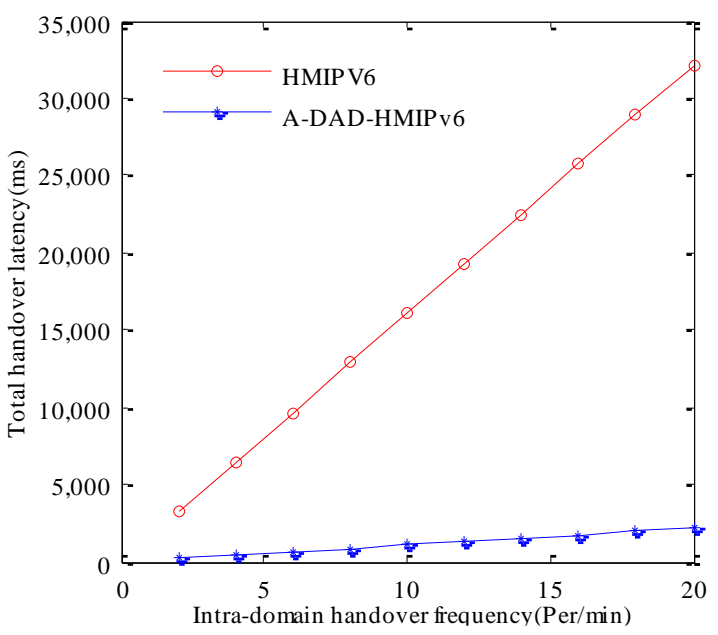

Figure 7. Results of intra-domain handover 


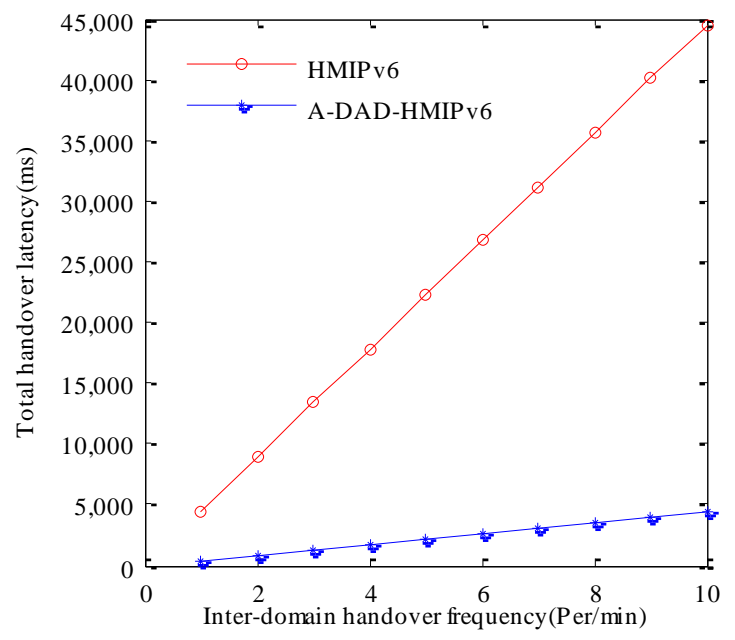

Figure 8. Results of inter-domain handover.

\section{Discussion}

A-DAD-HMIPv6 reduces the duration of a handover, and consequently it also reduces the packet loss during a handover. A-DAD-HMIPv6 is unlike previous proposals [4], [9], [10], [11], [12] require more messages or entities to the standard HMIPv6. A-DAD-HMIPv6 just needs to change the process of address configuration on MAP, so it could work with other MAPs which don't apply A-DAD-HMIPv6. Moreover, A-DAD-HMIPv6 needn't more supports of router or a unique IP address on internet. Therefore, it is easier to be implemented and programmed. Since it does not introduce any DAD operation, it also consumes less power during a handover comparing to other proposals.

\section{Conclusion}

Optimization algorithm is stared from address configuration aspects, by modifying the interface identifier structure and using the mechanism of address look-up table in advance to ensure that the interface identifier is unique in the MAP domain. Therefore MN is no longer needed DAD operation in the configuration process of new address, so that handover latency of $\mathrm{MN}$ can be reduced. This optimization algorithm of HMIPv6 doesn't appear in the prior. Mathematical results show that, in the case of $\mathrm{MN}$ moves relatively high frequency, A-DAD-HMIPv6 can still remain fairly low and stable handover latency. Therefore, we can design a prototype program supporting high QoS service mobility management through the algorithm.

Currently, Mobile IP technology will be mainly used in car networking and Internet of things, so the frequency handover is very common. A-DAD-HMIPv6 can be adapted to the application environment well and it can effectively reduce the handover latency.

\section{ACKNOWLEDGMENTS}

This work was supported in part by National Natural Science Foundation of China under grant 61402526, 61402525 and 61502528 , and in part by National High Technology Research and Development Program of China under grant 2012AA012902.

\section{REFERENCES}

[1] Johnson, D., Perkins, C., Arkko, J.: Mobility support in IPv6. S. IETF RFC 3775 (2004)

[2] 2. Soliman, H., EIMaki, H., Bellier, L.: Hierarchical mobile IPv6 mobility management (HMIPv6). S. IETF RFC 4140 (2005)

[3] Han, Y.H., Jeong, D.: A comprehensive study on handover performance of hierarchical mobile IPv6. J. Embedded and Ubiquitous computing. 2006, 1108-1118 (2006)

[4] Jung, H.Y., Soliman, H., Koh, S.J.: Fast handover for hierarchical MIPv6 (F-HMIPv6). In: The 6th international Conference on Advanced Communication Technology, pp. 551--554. IEEE Press, Ganwon-Do (2004)

[5] Rajeev, K.: Fast handovers for mobile IPv6. S. IETF RFC 4068 (2005)

[6] Dutta, N., Misra, I.S.: Multilayer hierarchical model for mobility management in IPv6: A mathematical exploration. J. Wireless Personal Communications. 78(2), 1413-1439 (2014)

[7] Abdullah., Radhwan, M., Zukarnain.: Networks seamless mobility support in heterogeneous wireless networks. J. International Journal of Advancements in Computing Technology. 7(3), 69-78 (2015)

[8] Song, M., Cho, J.D., Jeong, J.: On mathematical model of hierarchical mobility management protocols. In: Proceedings of the fourth international conference on Digital Information and Communication Technology and it's Applications(DICTAP), pp. 75-80. IEEE Press, Bangkok (2014)

[9] Yan, Z., Lee, X., Park, Y.J.: Enhanced HMIPv6 with cascaded tunnel. In: The 25th Annual International Symposium on Personal, Indoor, and Mobile Radio Communication (PIMRC), pp. 2199-2203. IEEE Press, Washington, DC (2014)

[10] Kaduic, E., Hadialic, M., Behlilovic, N.: Euclidean distance-based QoS metric for dynamic MAP selection in HMIPv6 networks. In: The 2015 IEEE International Conference on Computer and Information Technology; Ubiquitous Computing and Communications; Dependable, Autonomic and Secure Computing; Pervasive Intelligence and Computing (CIT/IUCC/DASC/PICOM), pp. 954-958. IEEE Press, Livepool (2015)

[11] Rana, M.K., Mandal, S., Sardar, B.: Optimized HMIPv6 (O-HMIPv6):reducing handoff latency in HMIPv6 networks. In: Applications and Innovations in Mobile Computing (AIMoC), pp. 18-24. IEEE Press, Kolkata (2015)

[12] Hong, Y.X., Pawlikowski, K., Sirisena, H.: DAD-Less MIPv6 for Reduced Handover Latency. In: International Conference on Innovative Mobile \& Internet Services in Ubiquitous Computing, pp. 353-360. IEEE Press, Seoul, (2011)

[13] Vogt, C.: A comprehensive and efficient handoff procedure for IPv6 mobility support. In: The 2006 International Symposium on the World of Wireless, Mobile and Multimedia Networks, pp. 212-218. IEEE Press, Washington, DC (2006) 